\title{
Procesamiento alimenticio y tecnológico de guanacos (Lama guanicoe) durante el Holoceno tardío: el caso del sitio costero Marazzi 32 (Tierra del Fuego, Chile)
}

\author{
Víctor Sierpe, Flavia Morello, Mauricio Massone y Cristóbal Palacios \\ Recibido 16 de abril 2019. Aceptado 26 de julio 2019
}

\begin{abstract}
RESUMEN
Presentamos el análisis del conjunto de guanacos provenientes del sitio arqueológico Marazzi 32, Isla Grande de Tierra del Fuego. El yacimiento, con dataciones entre 560-635 años AP, destaca por un registro óseo de cetáceos, mayoritariamente localizados en superficie, y la predominancia de guanaco en relación con otros mamíferos marinos, aves y otras especies. Los resultados permitieron confirmar la importancia de los restos de guanaco en la subsistencia y en el aprovisionamiento de materias primas óseas, lo que apoya la teoría económica que tradicionalmente ha considerado que los recursos terrestres jugaron un rol fundamental para los grupos humanos durante el Holoceno tardío. Se discute el interés de la explotación de guanacos en el sitio, el aprovechamiento mayoritario de individuos juveniles y las estrategias de explotación, cadena alimentaria y la gestión tecnológica de la especie.
\end{abstract}

Palabras clave: Zooarqueología; Cazadores-recolectores; Explotación; Instrumental óseo, Patagonia.

\begin{abstract}
ALIMENTARY AND TECHNOLOGICAL PROCESSING OF GUANACOS (LAMA GUANICOE) DURING THE LATE HOLOCENE: THE CASE OF COASTAL SITE MARAZZI 32 (TIERRA DEL FUEGO, CHILE). The analysis of the guanaco assemblage recovered from Marazzi 32 archaeological site, Isla Grande de Tierra del Fuego, is presented. The site, dated between 560 and 635 BP, is noteworthy for its record of cetacean bones, mostly found on the surface, and the prevalence of guanacos in relation to other marine mammals, birds and taxa. Results confirmed the importance of guanaco remains in the subsistence and supply of bone raw material, supporting the economic theory that has traditionally considered that land resources played a fundamental role for human groups during the late Holocene. The significance of guanaco exploitation at the site, the greater number of young individuals, and the exploitation strategies, food chain, and technological management of the species are discussed.
\end{abstract}

Keywords: Zooarchaeology; Terrestrial hunter-gatherers; Exploitation; Bone tools; Patagonia.

\footnotetext{
Víctor Sierpe. Programa de Doctorado UTA/UCN Universidad de Tarapacá, 18 de Septiembre 2222, Arica, Chile. Centro de Estudios del Hombre Austral, Instituto de la Patagonia, Universidad de Magallanes, Avenida Bulnes 0I890, Punta Arenas, Chile. E-mail: victor.sierpe@umag.cl

Flavia Morello. Centro de Estudios del Hombre Austral, Instituto de la Patagonia, Universidad de Magallanes, Avenida Bulnes 01890, Punta Arenas, Chile. E-mail: flavia.morello@umag.cl

Mauricio Massone. Centro de Estudios del Hombre Austral, Instituto de la Patagonia, Universidad de Magallanes, Avenida Bulnes 01890, Punta Arenas, Chile. E-mail: mauricio.massone@gmail.com

Cristobal Palacios. Centro de Estudios del Hombre Austral, Instituto de la Patagonia, Universidad de Magallanes, Avenida Bulnes 01890, Punta Arenas, Chile. E-mail: cristobal.palacios@umag.cl
} 


\section{INTRODUCCIÓN}

La importancia económica del guanaco (Lama guanicoe) entre los cazadores-recolectores del Holoceno en Tierra del Fuego se evidencia en el abundante y constante registro de la especie, reconocida como su principal recurso alimenticio terrestre (Casamiquela 1983; Mengoni Goñalons 1983, 1999; Borrero 1985, 2013; Borrero y Franco 1997; De Nigris 2004; Muñoz 2000, 2002, 2012, entre otros). Respecto de las sociedades cazadorasrecolectoras pedestres de la isla, datos etnográficos del siglo XIX muestran un aprovechamiento completo del cuerpo del animal, ligado no solo al uso de los huesos, sino también a los tendones, piel y otros subproductos orgánicos (Gusinde 1982; Chapman 1986). Además, su presencia en los yacimientos arqueológicos ha impulsado el desarrollo de variadas líneas de investigación interpretativas sobre la explotación de la especie como alimento y materia prima para la confección de instrumental de hueso.

Los estudios sobre la explotación para el consumo alimenticio del guanaco han estado fuertemente desarrollados en la región patagónica, lo que permite discutir aspectos metodológicos e impulsar el desarrollo de líneas interpretativas relativas a la adquisición y el consumo de la especie (e.g., Borrero 1990; Muñoz 1997, 2000, 2008; Mengoni Goñalons 1999; Mengoni Goñalons y De Nigris 1999; De Nigris 2004; De Nigris y Mengoni Goñalons 2004; De Nigris y Catá 2005; Otaola y Franco 2008; Alunni y Zangrando 2012; San Román et al. 2014, entre otros).

Con respecto al instrumental de hueso de guanaco, diversos trabajos han mostrado la representación de estas piezas en diferentes contextos de Tierra del Fuego (Massone 1988; Fiore 2011, 2012; Santiago et al. 2019, entre otros). En tanto, la clasificación y discusión sobre el uso de materias primas óseas ha sido desarrollada por Scheinsohn (1993-1994, 1997, 2010, 2013) y recientemente por Christensen (2016), quien ha profundizado los estudios sobre el uso de estas materias e identificó los estigmas asociados a los diferentes procesos de manufactura, y de esta manera logró recrear las diferentes cadenas operativas.

Este trabajo tiene como objetivo identificar las principales modificaciones antrópicas dentro del conjunto óseo de guanacos del sitio Marazzi 32 (MA-32), poniendo en perspectiva la explotación de la especie y un primer avance en la investigación sobre la tecnología ósea en el sitio.

\section{Caso de estudio}

El sitio Marazzi 32 (MA-32) está ubicado en bahía Inútil, próximo a la desembocadura del río Marazzi (norte de Tierra del Fuego, Chile) (Figura 1). Corresponde a uno de varios registros costeros a cielo abierto y de tiempos tardíos (Massone et al. 2003; Massone y Morello 2007; Massone 2017), con fechados de entre 670 y 560 años AP (Tabla 1). Al inicio de las investigaciones se identificaron en superficie seis conjuntos con restos óseos de cetáceos depositados sobre una berma de playa (Borella 2003), los cuales se extienden junto con otros restos de fauna por una superficie aproximada de $300 \mathrm{~m}^{2}$ (Massone y Morello 2007).

Las diferentes campañas de excavación (20022004) permitieron la recolección sistemática de material arqueológico en tres unidades, denominadas $16 \mathrm{D}, 16 \mathrm{E}$ y $13 \mathrm{H}$ (de $2 \times 2 \mathrm{~m}$ ) y delimitadas por sectores (1, 2, 3 y 4) (Massone y Morello 2007). Entre los materiales líticos hallados allí se encuentran raspadores y grandes rodados astillados con filos naturales amplios (Massone y Morello 2007). Destacan en la arqueofauna gran cantidad de restos de guanaco $y$, en menor medida, otras especies de mamíferos terrestres y marítimos, además de aves, peces $^{1}$ y escasos restos malacológicos.

\section{MATERIALES Y MÉTODOS}

En el presente trabajo se entregan los resultados del análisis de los restos óseos de guanaco

\begin{tabular}{|l|c|c|c|c|c|c|}
\hline Material & Unidad & $\begin{array}{c}\text { Nivel y } \\
\text { profundidad } \\
\text { muestra }\end{array}$ & Otros & Laboratorio & Años AP & $\begin{array}{c}\text { Cal. AP } \\
\text { (ShCal 13) } \\
(\boldsymbol{p = . 9 5})^{*}\end{array}$ \\
\hline Carbón & $13 \mathrm{H}$ & $\begin{array}{c}\text { Estrato II } \\
(10-20 \mathrm{~cm}) \\
20-22 \mathrm{~cm}\end{array}$ & Fogón 16 & Beta-201495 & $670 \pm 100$ & $741-495$ \\
\hline Carbón & $16 \mathrm{E}$ & $\begin{array}{c}\text { Estrato I } \\
(0-10 \mathrm{~cm}) \\
5-10 \mathrm{~cm}\end{array}$ & Fogón 18 & Ua-21185 & $560 \pm 35$ & $624-500$ \\
\hline Carbón & $16 \mathrm{E}$ & $\begin{array}{c}\text { Estrato I } \\
(0-10 \mathrm{~cm}) \\
5-10 \mathrm{~cm}\end{array}$ & Fogón 17 & Ua-21184 & $635 \pm 35$ & $651-536$ \\
\hline
\end{tabular}

Nota: * Fechados radiocarbónicos calibrados a 2 sigma con el programa OxCal 4.3 v4.3.2 (Bronk Ramsey 2017); r.5: SHCal13 atmospheric curve (Hogg et al. 2013).

Tabla 1. Fechados radiocarbónicos disponibles para MA-32 (Massone y Morello 2007). 


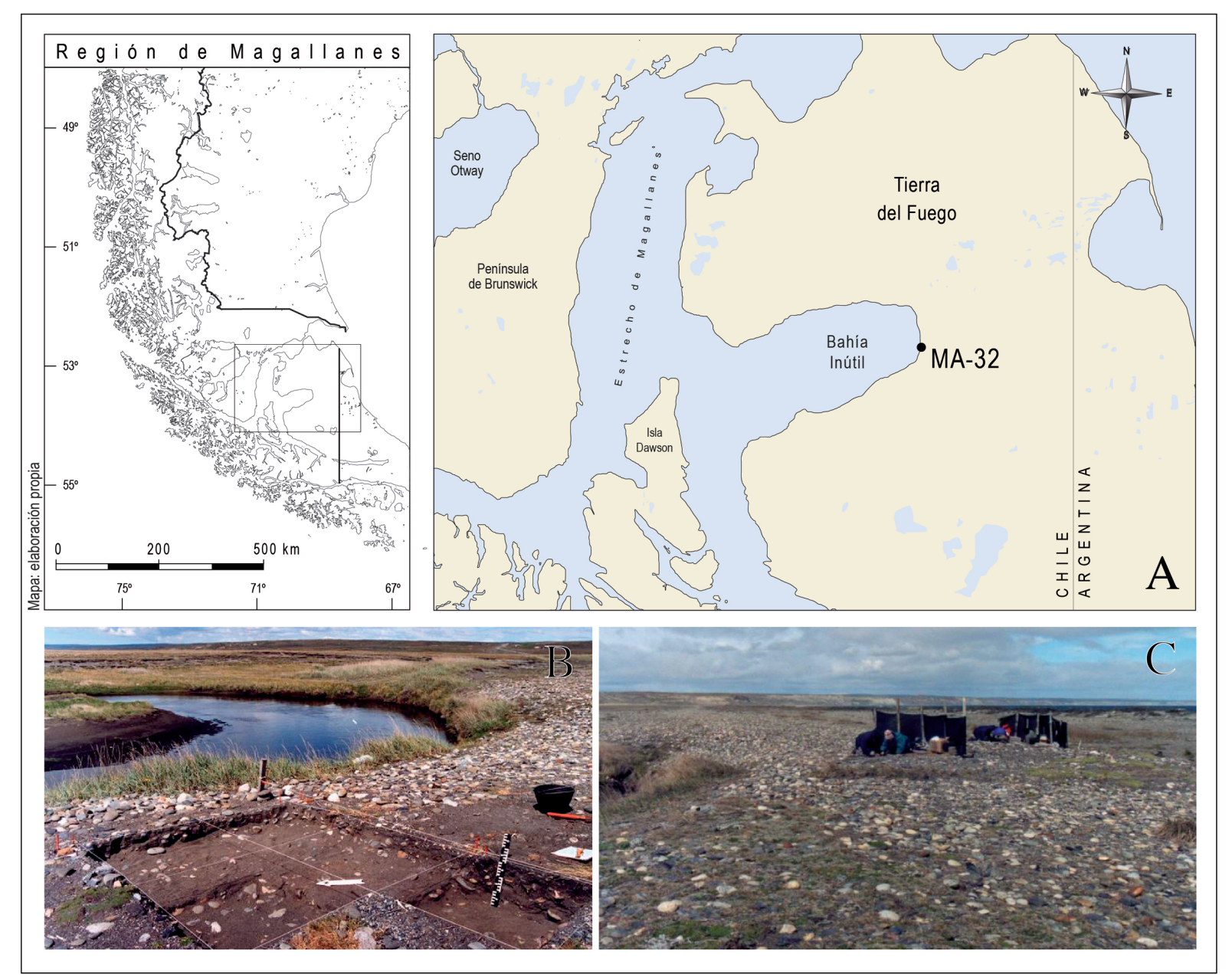

Figura 1. A. Mapa de ubicación sitio MA-32; B. Unidades de excavación 16E y 16D, vista este; C. Panorámica sitio MA-32, vista norte (Fotografías Mauricio Massone).

provenientes de las unidades de excavación 16E, $16 \mathrm{D}$ y $13 \mathrm{H}$, las cuales suman $12 \mathrm{~m}^{2}$ y totalizan 2710 restos atribuidos a la especie. Para la determinación anatómica y taxonómica se compararon los restos con material de referencia en los laboratorios del Centro de Estudios del Hombre Austral ${ }^{2}$ y un manual osteológico de la especie (Sierpe 2015). Se utilizaron los siguientes criterios de cuantificación: NISP (Lyman 2008), MNI (Poplin 1976; Lyman 2008), MNE (Binford 1978, 1981; Bunn 1982) y MAU de Lymam (1994) para la representación de cada unidad anatómica en la muestra arqueológica. La estimación de la edad fue calculada por medio de la fusión ósea a partir de los criterios presentados por Kaufmann (2009).

Los análisis cualitativos consideraron alteraciones tafonómicas de origen animal (perforaciones, agujeros, surcos, arrastre); y de origen biótico y abiótico, incluyendo alteraciones químicas, raíces, pisoteo y meteorización; por su parte, se consideraron modificaciones de origen cultural como huellas de corte, fractura y acción térmica (Parker y Toots
1970; Behrensmeyer 1978, 1990; Berner 1981; Binford 1981; Lyman 1994; Patou-Mathis 1994).

Para el análisis de la tecnología ósea, entre los estigmas presentes se consideraron puntos de impacto, ranurado, plano de extracción y abrasión, bajo la propuesta realizada por Christensen (2016) para los procesos de modificación tecnológica.

\section{RESULTADOS}

El total de la fauna vertebrada registrada es de 9426 restos (Tabla 2). Entre los más numerosos se encuentra Lama guanicoe, con 2710 (28,75\%) (Tabla 3); seguido por restos indeterminados adscritos a la Clase Aves, con 2624 restos (27,84\%); y a la Clase Mammalia, con 2393 (25,39\%) (Figura 2). Entre los restos adscritos a cada clase, el \%NISP corresponde a 55,78\% para Mammalia, 31,03\% para Aves y $13,19 \%$ para Actinopterygii. 


\begin{tabular}{|l|c|c|}
\hline \multicolumn{1}{|c|}{ Taxón } & NISP & \%NISP \\
\hline Lama guanicoe & 2710 & 28,75 \\
\hline Ovis aries & 4 & 0,04 \\
\hline Lycalopex culpaeus & 4 & 0,04 \\
\hline Canidae indet. & 5 & 0,05 \\
\hline Rodentia indet. & 17 & 0,18 \\
\hline Arctocephalus australis & 2 & 0,02 \\
\hline Otaria flavescens & 4 & 0,04 \\
\hline Otariidae indet. & 8 & 0,08 \\
\hline Mirounga leonina & 2 & 0,02 \\
\hline Cfr. Phocidae & 1 & 0,01 \\
\hline Pinnipedia indet. & 10 & 0,11 \\
\hline Cetacea indet. & 88 & 0,93 \\
\hline Delphinidae indet. & 10 & 0,11 \\
\hline Mammalia indet. & 2393 & 25,39 \\
\hline Chloephaga sp. & 3 & 0,03 \\
\hline Anatidae indet. & 12 & 0,13 \\
\hline Charadriiformes indet. & 5 & 0,05 \\
\hline Phalacrocorax sp. & 12 & 0,13 \\
\hline Diomedeidae indet. & 138 & 1,46 \\
\hline Procellariiformes indet. & 79 & 0,84 \\
\hline Passeriformes indet. & 13 & 0,14 \\
\hline Strigiformes indet. & 1 & 0,01 \\
\hline Aptenodytes patagonicus & 6 & 0,06 \\
\hline Aptenodytes cfr. forsteri & 1 & 0,01 \\
\hline Spheniscidae indet. & 31 & 0,33 \\
\hline Aves indet. & 2624 & 27,84 \\
\hline Odontesthes sp. & 597 & 6,33 \\
\hline Eleginops maclovinus & 566 & 6,00 \\
\hline Austrolycus sp. & 28 & 0,30 \\
\hline Merluccius sp. & 32 & 0,34 \\
\hline Salilota australis & 2 & 0,02 \\
\hline Serranus capensis & 17 & 0,18 \\
\hline Thyrsites atun & 1 & 0,01 \\
\hline Total & $\mathbf{9 4 2 6}$ & $\mathbf{1 0 0}$ \\
\hline
\end{tabular}

Tabla 2. Representación taxonómica en MA-32.

\section{Estrategias de explotación,} cadena alimentaria y tratamiento de guanacos en el sitio

La elección de presas se ve representada por un NMI de $L$. guanicoe de 18 individuos. $^{3}$ Del total de los restos atribuidos a la especie, solo $37(1,36 \%)$ se presentan completamente fusionados; entre ellos, han sido identificados en el conjunto tres individuos adultos. Sobre la estimación de edad, los estados de fusión ósea nos permitieron separar un mínimo de 15 individuos con una edad promedio de un año (877 restos, correspondientes al 32,36\%). El

\begin{tabular}{|c|c|c|c|c|c|c|c|}
\hline Elemento & NR & $\begin{array}{c}\text { MNE } \\
\text { izquierdo }\end{array}$ & $\begin{array}{c}\text { MNE } \\
\text { derecho }\end{array}$ & $\begin{array}{l}\text { MNE } \\
\text { axial }\end{array}$ & MAU & $\%$ MAU & MNI \\
\hline Cráneo & 86 & - & - & 3 & 3 & 9,7 & 3 \\
\hline Dientes sueltos & 82 & - & - & 28 & 5 & 16,1 & 5 \\
\hline Mandíbula & 53 & 10 & 5 & - & 7,5 & 24,2 & 10 \\
\hline Atlas & 35 & - & - & 18 & 18 & 58,1 & 18 \\
\hline Axis & 17 & - & - & 9 & 9 & 29 & 9 \\
\hline Vértebra cervical & 107 & - & - & 37 & 7,4 & 23,9 & 8 \\
\hline Vértebra torácica & 60 & - & - & 10 & 1,2 & 3,9 & 8 \\
\hline Vértebra lumbar & 82 & - & - & 67 & 5,2 & 16,8 & 6 \\
\hline Sacro & 1 & - & - & 1 & 1 & 3,2 & 1 \\
\hline Vértebra caudal & 8 & - & - & 8 & 2,5 & 8,1 & 1 \\
\hline $\begin{array}{l}\text { Vértebra } \\
\text { indeterminada }\end{array}$ & 739 & - & - & 18 & - & , & - \\
\hline Costilla & 212 & 39 & 25 & - & 4,5 & 14,5 & 4 \\
\hline Esternebra & 6 & - & - & 6 & 6 & 19,4 & 2 \\
\hline Escápula & 51 & 12 & 6 & - & 9 & 29 & 12 \\
\hline Húmero & 99 & 17 & 7 & - & 12 & 38,7 & 17 \\
\hline Radio & 80 & 26 & 36 & - & 31 & 100 & 14 \\
\hline Ulna & 22 & 8 & 13 & - & 10,5 & 33,9 & 11 \\
\hline Radio-Ulna & 24 & 7 & 10 & - & 85 & 27,4 & 5 \\
\hline Escafoide & 18 & 9 & 9 & - & 9 & 23 & 9 \\
\hline Lunar & 13 & 6 & 7 & - & 6,5 & 20,9 & 7 \\
\hline Piramidal & 14 & 4 & 10 & - & 7 & 22,6 & 10 \\
\hline Trapezoide & 5 & 3 & 2 & - & 2,5 & 8,1 & 3 \\
\hline Magnum & 8 & 4 & 4 & - & 4 & 12,3 & 4 \\
\hline Unciforme & 14 & 7 & 7 & - & 7 & 2,6 & 7 \\
\hline Pisiforme & 6 & 5 & 1 & - & 3 & 9,67 & 1 \\
\hline Sesamoideo & 11 & - & 9 & - & 0,7 & 2,2 & 2 \\
\hline Metacarpo & 19 & 8 & 8 & - & 8 & 25,8 & 4 \\
\hline Pelvis & 56 & 8 & 10 & - & 9 & 29 & 10 \\
\hline Fémur & 81 & 28 & 31 & - & 29,5 & 95,16 & 16 \\
\hline Patella & 3 & 2 & 1 & - & 1,5 & 4,8 & 2 \\
\hline Tibia & 91 & 40 & 42 & - & 17 & 54,8 & 13 \\
\hline Astrágalo & 22 & 13 & 8 & - & 10,5 & 33,9 & 13 \\
\hline Calcáneo & 26 & 10 & 10 & - & 10 & 32,3 & 10 \\
\hline Maleolo & 18 & 9 & 9 & - & 9 & 29 & 9 \\
\hline Navicular & 15 & 7 & 10 & - & 8,5 & 27,4 & 10 \\
\hline $\begin{array}{l}\text { Cuneiforme } \\
\text { menor }\end{array}$ & 2 & 0 & 2 & - & 1 & 3,22 & 2 \\
\hline Entocuneiforme & 9 & 5 & 4 & - & 4,5 & 14,5 & 5 \\
\hline Cuboide & 14 & 7 & 7 & - & 7 & 22,5 & 7 \\
\hline Metatarso & 20 & 4 & 7 & - & 5,5 & 17,7 & 4 \\
\hline Falange 1 & 127 & - & - & 25 & 3,1 & 10 & 4 \\
\hline Falange 2 & 65 & - & - & 11 & 1,4 & 4,5 & 2 \\
\hline Falange 3 & 6 & - & - & 4 & 0,5 & 1,6 & 1 \\
\hline Metapodio & 283 & - & - & 15 & 3,8 & 12,3 & 4 \\
\hline TOTAL & 2710 & 298 & 300 & 260 & - & - & 18 \\
\hline
\end{tabular}

Nota: *Cálculo realizado a partir de la recomposición o ensamblaje de los restos.

Tabla 3. Representación anatómica de L. guanicoe en MA-32. 


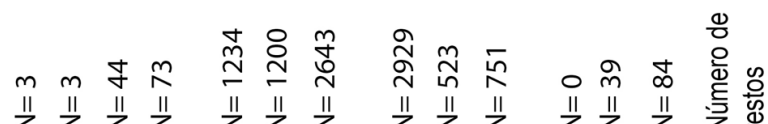

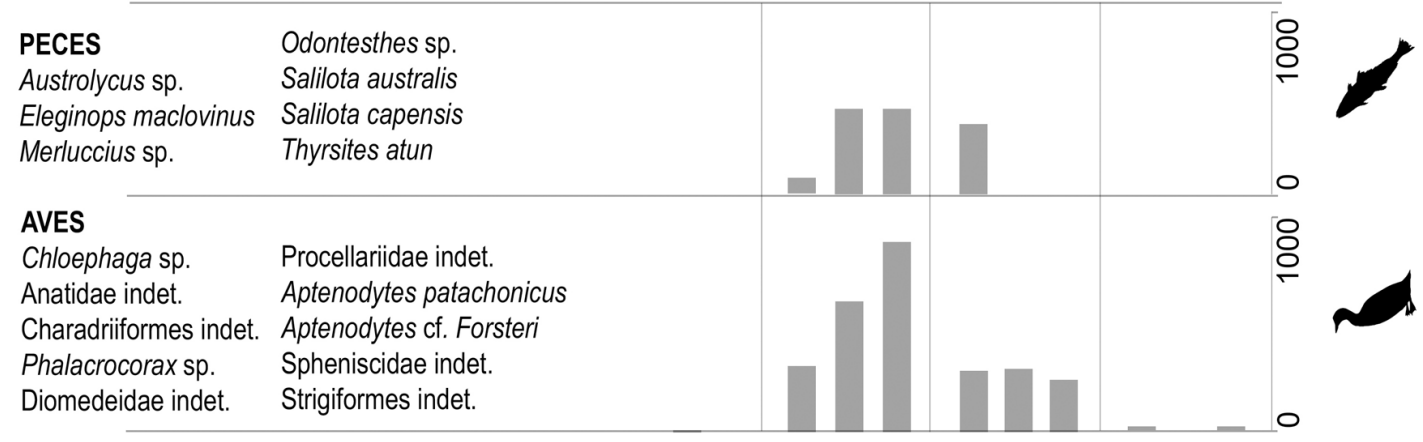

\section{PHOCIDAE}

Mirounga leonina

Phocidae indet.

OTARIIDAE

Arctocephalus australis

Otaria flavescens

Otariidae indet.

\section{PINNIPEDIA}

Pinnipedia indet.

\section{DELPHINIDAE}

Delphinidae indet.

\section{CETACEA}

Cetacea indet.

RODENTIA

Rodentia indet.

CANIDAE

Lycalopex culpaeus

Canidae indet.

Lama guanicoe

Artiodactyla indet.

ARTIODACTYLA

Ovis aries

MAMMALIA

Mammalia indet.
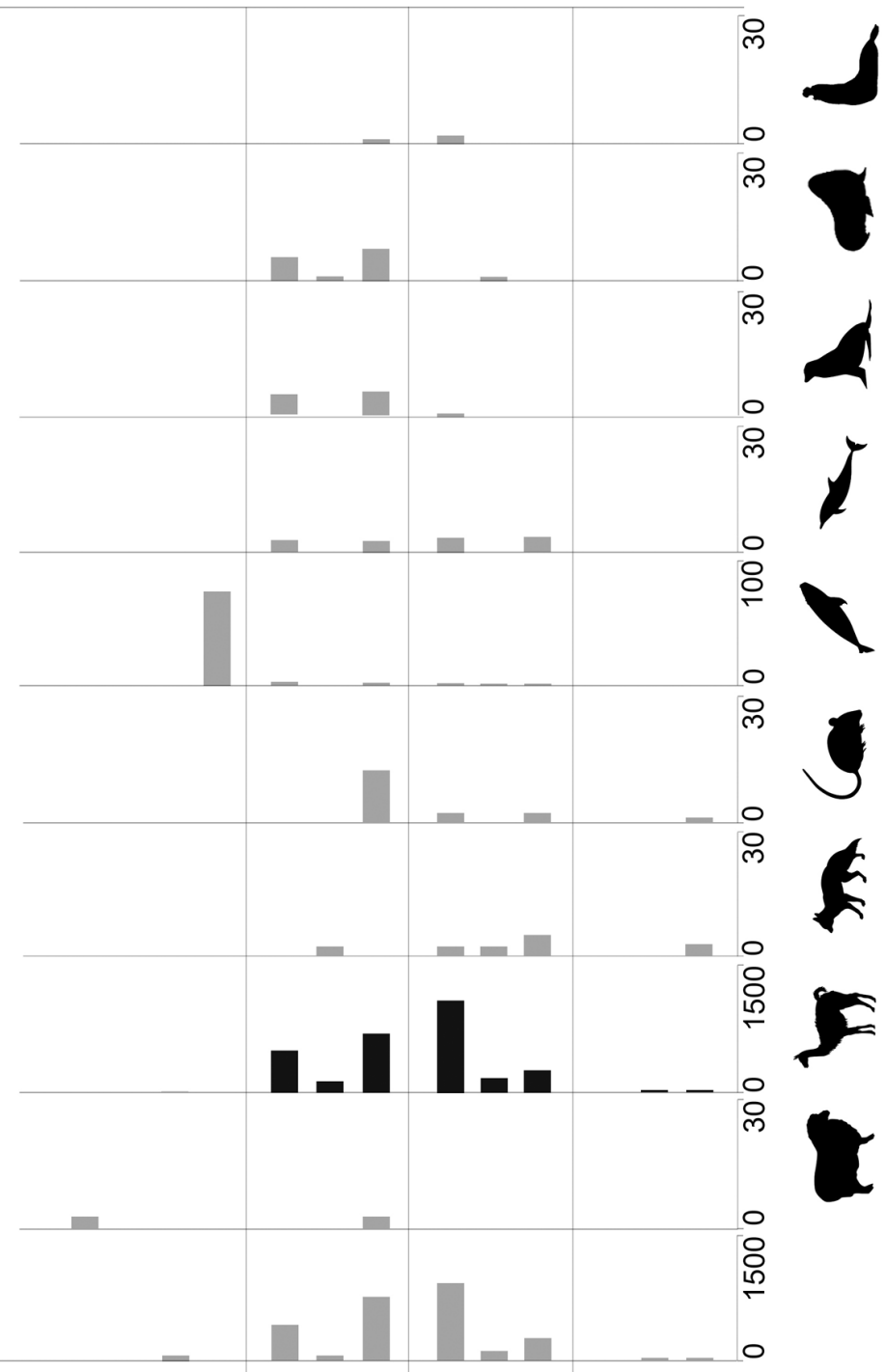

앙
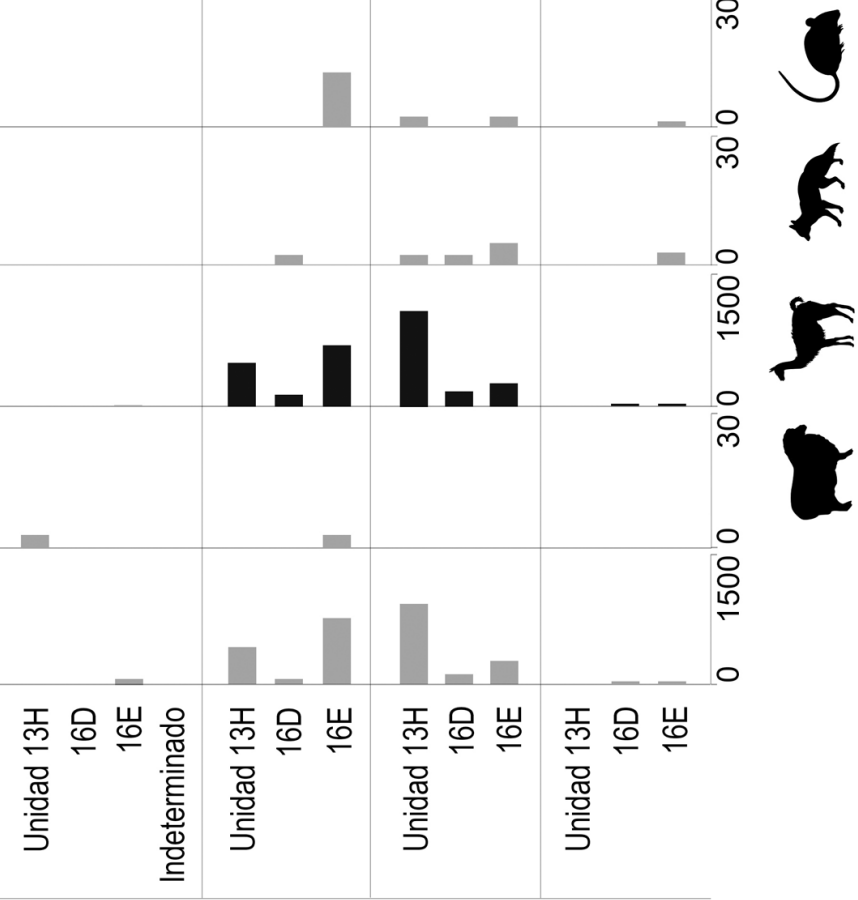

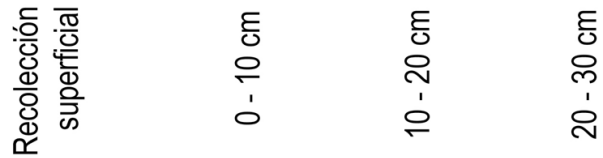


cálculo realizado sobre los restos de estos ejemplares ha considerado el ensamble completo de huesos largos sin fusión ósea, tomando en cuenta un cálculo mínimo de 5-6 meses a partir de las radio-ulnas, y 8-18 meses sobre los húmeros. En relación con la elección de presas entre machos o hembras, los restos no permitieron una identificación a esta escala dada la ausencia de elementos discriminatorios, como caninos (Raedeke 1978) o pelvis (De Lamo 1990; Gecele et al. 1996; Cartajena 2007).

Respecto de los procesos tafonómicos, los restos no presentan mayor alteración; son pocos los que están modificados por la acción de roedores $(n=40)$, termoalteración $(n=11)$, acción de carnívoros $(n=5)$, marcas de raíces $(n=6)$ o, finalmente, algún tipo de acción química $(n=2)$. Las alteraciones predominantes son por exposición a la intemperie (estadios 1, 2 y 3 sensu Behrensmeyer 1978), y afectaron principalmente a los restos de los estratos superiores o bien a restos óseos registrados en superficie (Tabla 4).

En relación con la cadena alimentaria del guanaco, el proceso de caza, búsqueda, persecución y muerte del animal pudo desarrollarse en proximidades del sitio, dado que la zona centro y norte de Tierra del Fuego es un territorio reconocido por la amplia distribución de guanacos que allí habitan, además de la acreditada explotación de la especie en la isla entre cazadores-recolectores a lo largo del Holoceno (Muñoz 2000; Calás 2009; Santiago 2013).

El transporte de animales completos se evidencia al registrar en el yacimiento la totalidad de los elementos anatómicos de la especie. La lectura de modificaciones antrópicas arrojó la presencia de huellas de corte en cráneo $(n=2)$, bajo la cresta sagital externa, metapodios $(n=2)$, sección medio-distal palmar (Figura 3a) y tibia $(n=1)$, sección distal, sobre la cóclea tibial, probablemente relacionadas con las etapas iniciales del procesamiento del animal. Elementos del esqueleto axial presentan huellas de corte asociadas a la desarticulación. Estas se hallan en la cintura pélvica $(n=2)$, cuerpo del ilion, vértebras torácicas $(n=$ $2)$, lumbares $(n=6)$ e indeterminadas $(n=2)$, preferentemente sobre y bajo apófisis transversa; costillas $(n=4)$, bajo la tuberosidad de la costilla y astrágalos $(n=6)$, sobre la sección medio-lateral y medial (bajo trócleas) (Figura 3b). En elementos del esqueleto apendicular las huellas se registran en radio-ulnas $(\mathrm{n}=3)$, en torno a la sección media de la incisura troclear (Figura 3c) y en la sección articular sobre la superficie articular del carpo y fémur $(n=1)$, bajo la línea intertrocantérica (Figura $3 d$ ). Huellas relacionadas con el descarne y la remoción de tejidos blandos fueron registradas en escápulas $(n=4)$, bajo la tuberosidad de la espina escapular; húmeros $(\mathrm{n}=2)$, en torno a la cara lateral y medial

\begin{tabular}{|c|c|c|c|c|c|c|c|c|c|}
\hline Estadios & Rec. sup. & $\%$ & Niv. 0-10 & $\%$ & Niv. 10-20 & $\%$ & Niv. 20-30 & $\%$ & N Totales \\
\hline 0 & 1 & 0,833 & 2110 & 88,1 & 2686 & 98,53 & 69 & 100 & 4866 \\
\hline 1 & 20 & 16,67 & 175 & 7,307 & 10 & 0,367 & 0 & 0 & 205 \\
\hline 2 & 83 & 69,17 & 84 & 3,507 & 3 & 0,11 & 0 & 0 & 170 \\
\hline 3 & 16 & 13,33 & 1 & 0,042 & 0 & 0 & 0 & 0 & 17 \\
\hline
\end{tabular}

Tabla 4. Relación de meteorización de restos óseos de mamíferos por nivel de excavación.
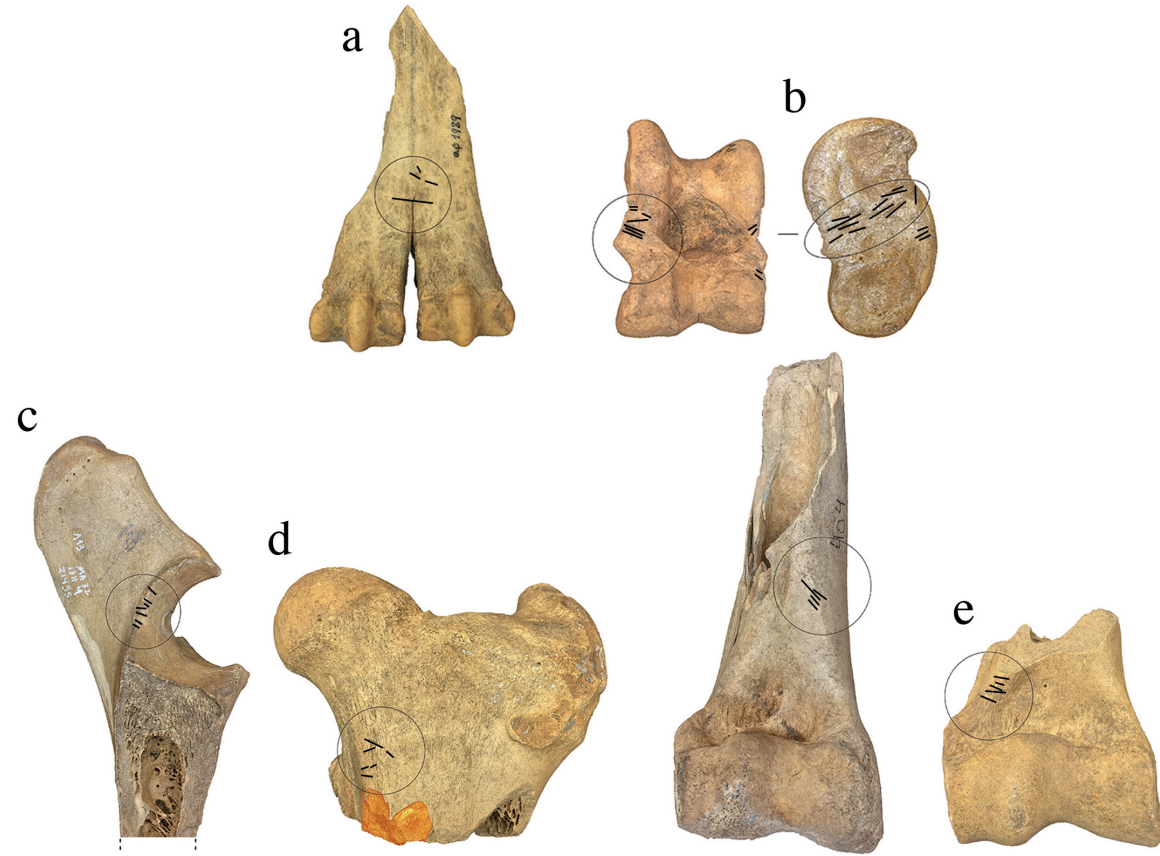

$0=-5 \mathrm{~cm}$

Figura 3. Huellas de corte registradas sobre: a. metapodio distal; b. astrágalos; c. epífisis proximal de radio-ulna; $d$. epífisis proximal de fémur -negativos de lascado en naranja-; e. epífisis distal de húmero. 
de la sección medio-distal (Figura 3e); vértebras cervicales $(n=4)$; sobre la base de apófisis transversa; y costillas $(n=1)$, sección lateral de la tuberosidad articular. Otras huellas fueron registradas sobre la sección media de falange primera $(n=1)$; tibia $(\mathrm{n}=1)$, sobre la sección latero-medial proximal; costillas $(n=5)$, sobre la sección medio-lateral y medial; y apófisis transversas aisladas $(n=1)$, sobre el cuerpo de la apófisis, lo que indica una relación con el procesamiento completo de los huesos (sensu Binford 1981; Muñoz 2000, 2012).

Entre los elementos anatómicos mejor representados del conjunto total se destaca la presencia de radio (mayor representatividad sobre elemento radio-ulna no fusionado con valor MAU del 100\%), fémur $(95,16 \% \mathrm{MAU})$, tibia $(54,8 \% \mathrm{MAU})$ y metapodios (metacarpo con 25,8\% MAU, metatarso con $17,7 \%$ MAU y metapodios con 12,3\% MAU); estos últimos con un alto porcentaje de fracturas $(44,54 \%$ para metapodios y $28,57 \%$ para tibias, sobre un total de 117 fracturas registradas). De los elementos anteriormente mencionados, un total de 37 restos $(31,1 \%)$ presentan puntos de impacto, registrados principalmente sobre tibias y metapodios. Destaca dentro del conjunto un metapodio distal modificado en su superficie por medio de un surco ranurado $(0,13 \%)$ (ver más adelante en resultados de análisis tecnológico).

Entre los tipos de fracturas registrados en huesos largos de individuos adultos $(\mathrm{n}=24)$, las más representativas son las fracturas en espiral (62,5\%), seguidas por longitudinales $(33,33 \%)$ y una indeterminada $(4,17 \%)$. En tanto en individuos juveniles $(n=93)$ se presenta un alto indice de fracturación, dominado por fracturas indeterminadas $(65,6 \%)$, seguidas por fracturas longitudinales (21,50\%), fracturas en espiral $(11,82 \%)$ y fracturas irregulares $(1,07 \%)$. En este conjunto es posible apreciar fragmentos proximales completos de metapodios (antero-posterior o, en este caso, también lateromedial, dada la no fusión ósea de estos) y fragmentos mediales con puntos de impacto lateral. Las fracturas, en su mayoría longitudinales y en espiral, evidencian la acción antrópica y el interés en la obtención de médula.

Finalmente se destacan ocho falanges primeras que comparten un patrón de fracturación posiblemente ligado al aprovechamiento de médula. La extracción de la sección medio-proximal del hueso carece de puntos de impacto, lo cual podría indicar que la fractura de las piezas fue realizada intencionalmente mediante la percusión desde el extremo medial o lateral de la falange.

\section{Análisis tecnológico}

Entre los restos analizados se registraron diez instrumentos óseos, dos de ellos confeccionados sobre diáfisis, y ocho piezas correspondientes a epífisis con modificación tipo machacador (sensu Hajduk y Lezcano 2005).

En el conjunto de elementos confeccionados sobre huesos largos de guanacos adultos se registra un fragmento medial de tibia, identificado como percutor blando/retocador (Figura 4a). La pieza presenta multiples estigmas, con modificaciones sobre ambas secciones, medio-proximal y medio-distal. La confección del elemento ha sido efectuada a partir de la modificación por medio de la extracción de los extremos proximal y distal del hueso -la ausencia de las epífisis limita a inferir el procedimiento de fracturación en esta etapa-. Sobre el cuerpo de la pieza se observan leves cúpulas de hundimiento $^{4}$ encima de la superficie dorsal proximal y en torno a toda la sección distal de esta, producto de lo cual aparecen estigmas de percusión sobre la superficie del hueso. Por su parte, se reconocen también huellas de uso y lascado sobre el borde distal lateral del hueso.

La segunda diáfisis modificada corresponde a la sección medio-craneal de un metapodio, el cual presenta abrasión en uno de sus extremos. La pieza ha sido identificada tipológicamente como espatuliforme (sensu Camps-Fabrer 1966) (Figura 4b).

En cuanto a las epífisis de huesos largos presentes en el conjunto, algunas de ellas fueron modificadas sobre la porción metafisiaria (Figura 4c y 4d). Estos artefactos están representados en ocho de las 19 epífisis presentes en el sitio (Tabla 5) (una sola coincide con guanaco juvenil) y se corresponden con los artefactos denominados previamente como machacadores óseos (Hajduk y Lezcano 2005).

La fracturación de huesos largos (sin patrones registrados) pudo estar enfocada en la extracción de médula, mientras que los restos proximales o distales más completos fueron retocados obteniendo planos transversales más o menos lineales y rectos. El desgaste de las piezas ha proporcionado un lascado continuo en torno al extremo medial del hueso, donde se encuentra el plano de extracción. La sección activa de todos los machacadores se 
a

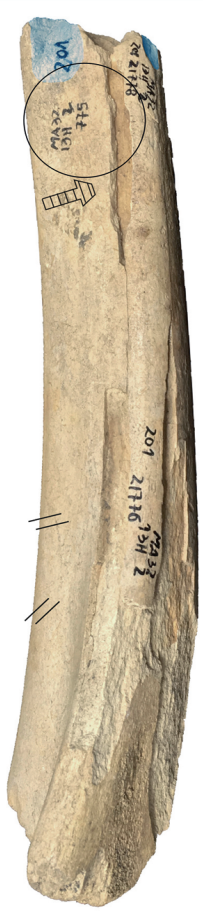

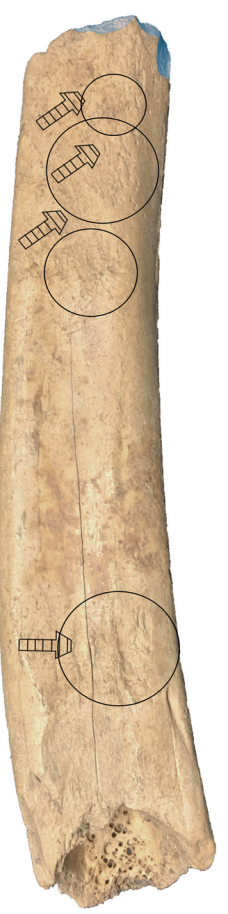

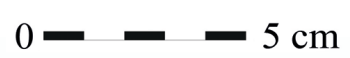

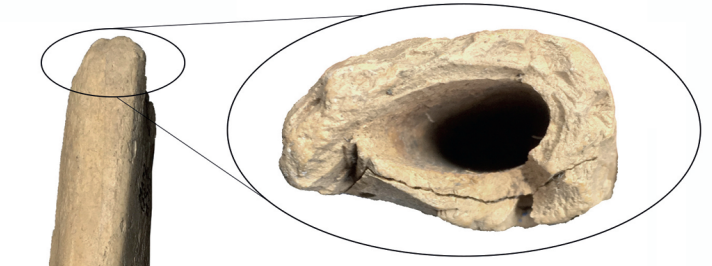

Extremo distal plano transversal

冒 Abrasión unidireccional
// Corte

b

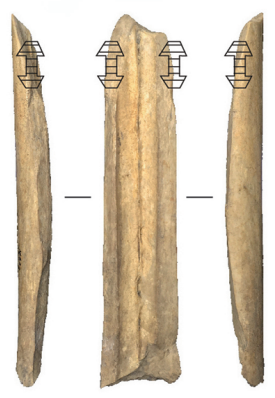

$0---5 \mathrm{~cm}$ c

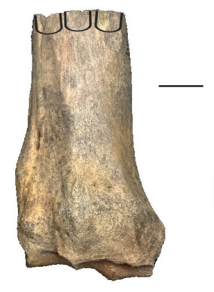

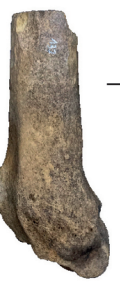

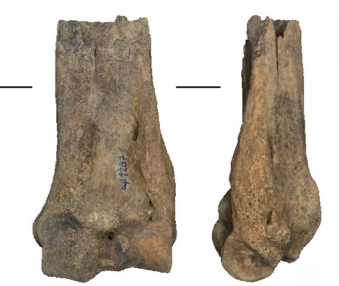

d

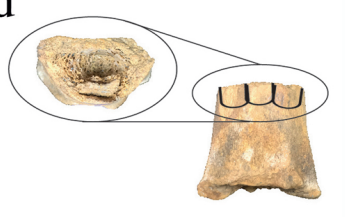

g

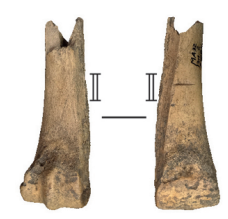

e

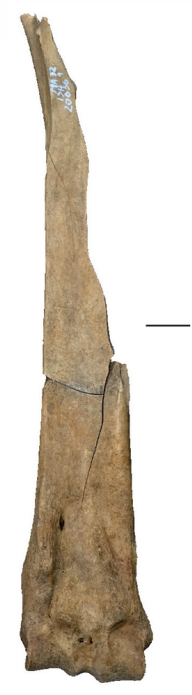

$0---5 \mathrm{~cm}$
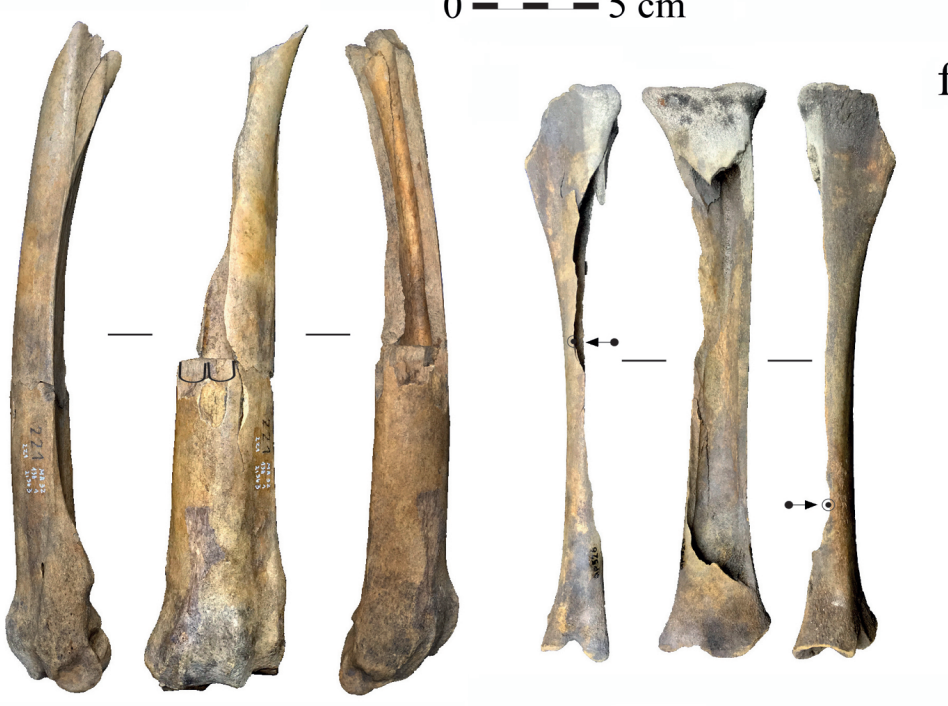

\section{f}

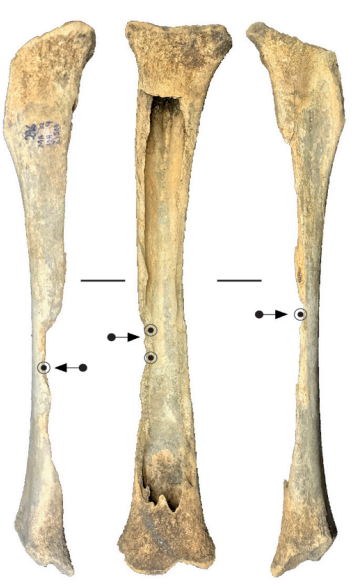

$0---5 \mathrm{~cm}$

- Percusión difusa $\odot$ Punto de impacto $\mathbb{I}$ Ranurado

弇 Confección/abrasión $\cap$ Plano de extracción

Figura 4. a. Percutor blando/retocador sobre tibia medial -en círculos cúpulas de hundimiento, en azul negativos de lascado-; b. espatuliforme sobre porción medial de metapodio; c. machacador sobre epífisis distal de radio-ulna; d. machacador sobre radio distal de individuo juvenil; e. fractura y obtención de machacador sobre radio-ulna medio distal; f. proceso de fracturación sobre porción medial de tibias de individuos juveniles; g. metapodio distal con ranurado sobre sección medial. 


\begin{tabular}{|c|c|c|c|c|c|c|}
\hline & \multirow{2}{*}{$\begin{array}{c}N^{0} \text { total } \\
\text { piezas }\end{array}$} & \multirow{2}{*}{$\begin{array}{l}\text { Epífisis con } \\
\text { planos de } \\
\text { extracción }\end{array}$} & \multicolumn{2}{|c|}{ Proximal } & \multicolumn{2}{|c|}{ Distal } \\
\hline & & & Izq. & Der. & Izq. & Der. \\
\hline Húmero & 7 & 2 & - & - & - & 2 \\
\hline $\begin{array}{l}\text { Radio- } \\
\text { Ulna } \\
\end{array}$ & 9 & 3 & - & - & 2 & 1 \\
\hline Fémur & 3 & 2 & 2 & - & - & - \\
\hline Tibia & 3 & 1 & - & - & - & 1 \\
\hline Total & 19 & 8 & & & & \\
\hline
\end{tabular}

Tabla 5. Representación de epífisis con modificación de tipo machacador en MA-32.

ubica sobre un plano transversal y la altura de esta se ve directamente relacionada con el producto de la fracturación, evidenciado en el presente caso por el ensamble de las piezas MA-32 21563 y MA-32 20656, donde se observa el trabajo de confección del machacador posterior a la fractura del hueso (Figura 4e).

Si bien no ha sido catalogado como instrumento, un nuevo elemento se destaca dentro del conjunto. Se trata de un metapodio fracturado en su porción medio-distal; este presenta ranurado longitudinal desde la fractura del hueso a la sección de fusión articular condilar distal (Figura 4g), a través del canal metacarpiano distal, longitudinalmente por las caras craneal y palmar del hueso. No se reconocen otras piezas con este tipo de estigma en el sitio.
Entre los guanacos juveniles, los únicos elementos anatómicos que presentan estigma de desbaste claro corresponden a tibias $(n=2)$ (Figura $4 f)$. Sin embargo, se desconoce si estas fracturas registradas provienen de la producción de artefactos, ya que en el sitio no se cuenta con instrumentos óseos que pudieran haber sido elaborados sobre la sección medial de tibias de guanacos juveniles. La escasa evidencia tecnológica sobre estos ha imposibilitado recrear procesos de desbaste y fabricación técnica a los cuales pudieron estar sometidos los restos óseos de guanacos juveniles.

Los resultados obtenidos han sido resumidos en un esquema de las cadenas operativas relevadas para el sitio MA-32 (Figura 5), y dan cuenta de la representatividad de huesos de guanaco y la selección de piezas para la confección de artefactos óseos registrados en el sitio.

\section{DISCUSIÓN Y CONCLUSIONES}

Los resultados obtenidos a partir del análisis zooarqueológico realizado en el sitio MA-32 han demostrado la importancia de la explotación del guanaco, y apoyan la teoría económica que

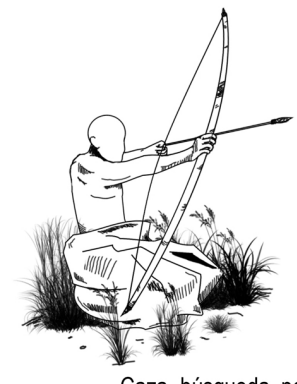

Caza -búsqueda, persecución y muerte del animalen proximidades del sitio
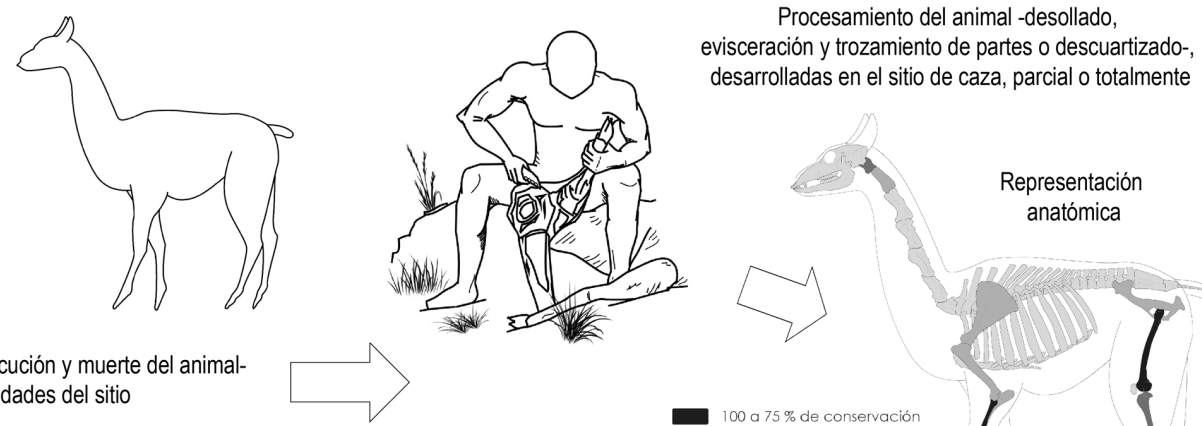
evisceración y trozamiento de partes o descuartizado-, rolladas en el sitio de caza, parcial o totalmente

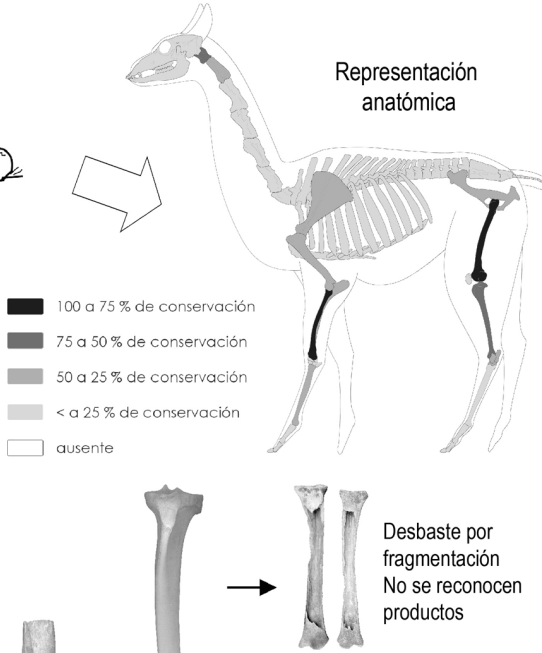

Alimentación: médula
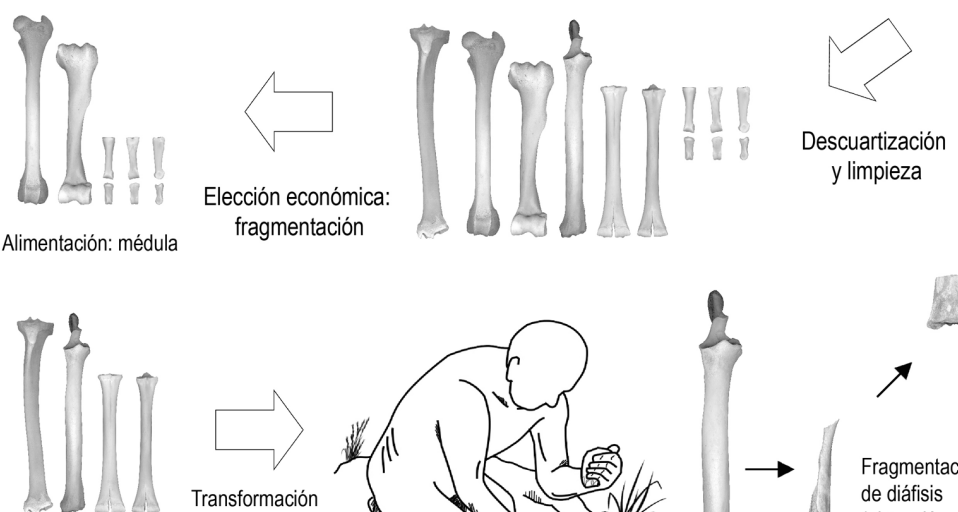

Alimentación: médula y uso Tecnológico
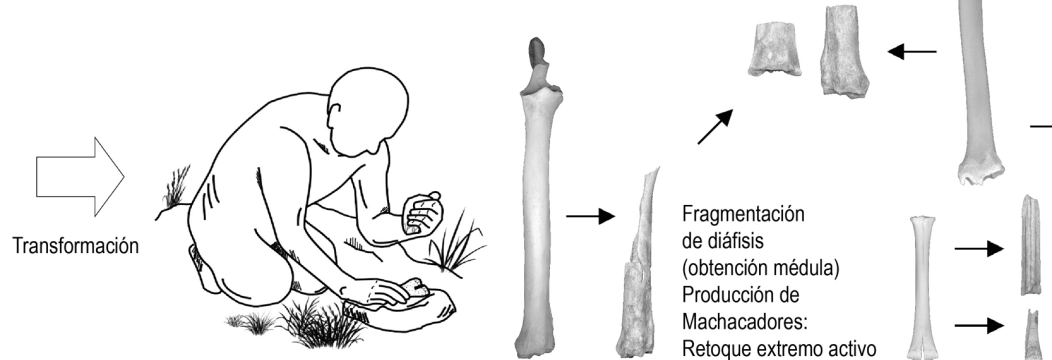

Desbaste por fragmentación Eliminación de epífisis Retoque. Producto: Percutor blando

Figura 5. Reconstrucción de la cadena operativa alimentaria y técnica de L. guanicoe en MA-32. 
sostiene la relevancia de los recursos terrestres para los grupos humanos que habitaron el norte de la isla de Tierra del Fuego durante el Holoceno tardío (Casamiquela 1983; Mengoni Goñalons 1983, 1999; Borrero 1985, 2013; Borrero y Franco 1997; Muñoz 2000, 2002; Prieto et al. 2007; Santiago 2013; Santiago y Salemme 2016, entre otros). Si bien este trabajo no constató el procesamiento de otros taxa registrados en el sitio, como ser otros mamíferos terrestres, mamíferos marinos, aves y otras especies, la alta representatividad y procesamiento de los restos de guanaco evidencia un aprovechamiento específico, tanto como recurso alimenticio, como de explotación de materia prima ósea para la fabricación de instrumental.

La representatividad de todos los elementos óseos del esqueleto del guanaco nos indica la incorporación de las carcasas completas al sitio. Huellas de corte y fracturas en huesos largos nos permiten presumir que estas fueron utilizadas de manera integral, y muestran también que el animal, además de aprovechado por su carne como alimento y por sus huesos como materia prima, fue explotado para el uso de cuero y tendones (e.g., huellas de corte sobre costillas y sobre sección palmar distal de metapodios). La fractura de huesos largos fue integral, y metapodios y tibias fueron los preferidos para la manofactura ósea (Christensen 2016; Sierpe 2018).

La alta representación de individuos juveniles de entre 8 y 18 meses de edad sugiere la caza y explotación de estos en periodo estival, coincidente con la etología de la especie, puesto que en dicha estación del año los machos adultos o, en este caso, juveniles solitarios, se agrupan (Franklin 1983). Esto pudo facilitar la obtención de los animales aquí representados, aunque, al no contar con la presencia de elementos diagnósticos de sexo entre los restos, no se descarta la presencia de hembras dentro del conjunto. Por su parte, restos adscritos a crías de entre 5 y 6 meses de edad coincidirían con la captura de grupos familiares, aunque la ausencia de estigmas antrópicos no permite descartar la incorporación natural de estos individuos debido a otros factores, como por ejemplo, estrés invernal (Raedeke 1978; Borrero 2001).

Se desconoce si la alta fragmentación en huesos largos de individuos juveniles -principalmente metapodios- proviene de la producción artefactual, dado que no se registaron piezas confeccionadas sobre estos huesos. De esta manera, y a partir del registro de escasos puntos de impacto y de la lectura de diáfisis con fractura longitudinal y en espiral, es posible suponer el aprovechamiento de estos elementos con un objetivo alimenticio y/o, además, plantear un ejercicio sobre la manufactura de piezas óseas más frágiles y en algunos casos, de menor tamaño. Este supuesto surge al comparar la representatividad de las tibias fracturadas de individuos juveniles, en las cuales todas las porciones medio-proximales comparten un proceso de fracturación, el que pudo estar ceñido bajo un patrón de extracción, registrado en dos piezas parcialmente completas. Es posible que el producto obtenido corresponda a un soporte largo y plano eventualmente bajo un objetivo técnico.

La presencia de machacadores óseos en variadas colecciones arqueofaunísticas ha sido largamente discutida en la arqueología patagónica (Silveira 1979; Bird 1988; Borrero 1994-1995; Muñoz y Belardi 1998; Mengoni Goñalons 1999; De Nigris 2004; Hajduk y Lezcano 2005; Santiago y Salemme 2016, entre otros). En su mayoría, estos evidencian el uso de partes proximales y distales de huesos largos de guanaco y fueron interpretados como de uso exclusivo de cazadores terrestres en la zona y como marcador de sus actividades (San Román 2004), aunque su registro también se ha encontrado en sitios de subsistencia mixta como Ponsonby, en isla Riesco (Legoupil 2003) y en otros de economía terrestre con incorporación ocasional de recursos costeros, como los sitios Las Vueltas (Santiago y Salemme 2016) y Heshkaia (Alunni 2018), en Tierra del Fuego. Por otra parte, también han sido reconocidos en otros elementos anatómicos con la misma morfología, tales como escápula, vértebra cervical y pelvis (Hajduk et al. 2004; Franco et al. 2007; Sierpe et al. 2009, entre otros). Además, pero en menor medida, los machacadores fueron elaborados sobre elementos óseos de otras especies, como ñandú (Caviglia y Borrero 1978), otáridos (Caracotche et al. 2005; Christensen 2016) y huemul (Sierpe et al. 2009). La evidencia de estas piezas en Tierra del Fuego -tanto para MA-32 como para otros sitios del norte de la isla, Marazzi 2 y Cabo Monmouth 20 (Sierpe 2018)- presume que la transmisión de su modo de ejecución y uso contó con la integración sociocultural de los pueblos canoeros en la zona. Por lo demás, las piezas no presentan modificaciones por corte, ranurado guía o el denominado "marcado perimetral", como se comprueba en localidades del centro norte de Argentina (e.g., Mengoni Goñalons y Silveira 1976; Silveira 1979; Acosta 2005; Loponte 2008, Acosta et al. 2014, 
entre otros). Esta diferenciación tecnológica responde al modelo de manufactura técnica de machacadores en la zona de Fuego-Patagonia (Sierpe 2018).

Respecto de la presencia de un metapodio ranurado, Christensen (2016) da cuenta de este proceso en otras colecciones de Fuego-Patagonia. La autora identifica esta técnica de desbaste en huesos de mamíferos terrestres asociada a la partición de metapodios de huemul y guanaco y, más puntualmente, durante los procedimientos de extracción sobre tibia en contextos del Holoceno tardío (Christensen 2016: 208).

Las observaciones registradas sobre los diferentes planos de la tibia medial identificada como percutor blando/retocador coindicen con los resultados presentados en trabajos experimentales sobre el uso de este elemento como retocador (Mallye et al. 2012; Mozota Holgueras 2012, 2015; Costamagno et al. 2017), en los que queda manifiesto que las huellas registradas pueden corresponder a estigmas producidos por el uso de este elemento vinculado a los procesos en la fabricación de industria lítica. Por su parte, Santiago et al. (2019) reconocen estigmas asociados al retoque por percusión blanda sobre la porción de un fragmento medial de tibia, en la que pudieron identificar microestrías y hoyuelos (definidos en el presente trabajo como "cúpulas de hundimiento").

Por último, podemos concluir que en el sitio MA-32 a fines del Holoceno en el norte de Tierra del Fuego, la explotación del guanaco estuvo marcada por la incorporación de las presas completas al yacimiento. Estas fueron aprovechadas de manera integral, tanto como alimento y en el uso de partes blandas, como para su empleo en procesos técnicos de manufacturación a partir de la materia prima ósea, lo que se evidencia en el uso de metapodios y tibias, y en el alto aprovechamiento de epífisis de huesos largos para su uso como machacadores óseos durante el período tardío.

\section{Agradecimientos}

Estudio financiado por la Dirección de Investigación, Postgrado y Transferencia Tecnológica, Universidad de Tarapacá, Chile, proyecto de Tesis de Investigación para Estudiantes de Postgrado titulado "Los artiodáctilos y su importancia económica entre los cazadores-recolectores del Holoceno medio y tardío de Fuego-Paragonia, Chile" -código 3729-17-; proyecto Fondecyt regular 1140939 "Arqueología de grandes Islas Fueguinas: Tierra del Fuego, Dawson y
Navarino, Poblamiento Humano e interacción cultural"; y Beca Nacional de Doctorado, convocatoria 2014, Comisión Nacional de Investigación Científica y Tecnológica CONICYT -Gobierno de Chile-. A Manuel San Román y Jimena Torres por discusiones emanadas durante los análisis; y a Dominique Legoupil y Marianne Christensen por comentarios durante la presentación del trabajo en las X Jornadas de Arqueología de la Patagonia, Puerto Madryn, 2017.

\section{REFERENCIAS CITADAS}

Acosta, A.

2005 Zooarqueología de cazadores-recolectores del extremo nororiental de la provincia de Buenos Aires (Humedal del Río Paraná Inferior, región Pampeana Argentina). Tesis Doctoral inédita. Facultad de Ciencias Naturales y Museo, Universidad Nacional de La Plata, La Plata.

Acosta, A., N. Buc y L. Mucciolo

2014 Elementos óseos con aserrado perimetral: el caso del Humedal del río Paraná inferior. Revista Chilena de Antropología 30 (2): 17-23.

Alunni, D. V.

2018 Estrategias de aprovisionamiento de guanacos y uso del bosque Magallánico por cazadores-recolectores costeros. Revista del Museo de Antropología 11 (2): $7-22$.

Alunni, D. V. y F. A. Zangrando

2012 Primeros datos sobre el transporte, procesamiento y consumo de guanacos en la localidad arqueológica Heshkaia (sudeste de Tierra del Fuego, Argentina). Magallania 40 (1): 319-331.

Behrensmeyer, A. K.

1978 Taphonomic and economic information from bone weathering. Paleobiology 4: 150-162.

1990 ICAZ Experimental Taphonomy Workshop: Workshop Outline and Notes. ICAZ: 1-17. Natural Museum of Natural History, Smithsonian Institution, Washington DC.

Berner, R. A.

1981 Authigenic mineral formation resulting from organic matter decomposition in modern sediments. Fortsher Miner 59 (1): 117-135.

Binford, L.

1978 Dimensional Analysis of Behavior and Site Structure: Learning from an Eskimo Hunting Stand. American Antiquity 43: 256-273. 
Binford, L.

1981 Bones: Ancient Men and Modern Myths. Academic Press, Nueva York.

Bird, J.

1988 Travels and Archaeology in South Chile. University of lowa Press, lowa.

Borella, F.

2003 Informe en relación a las concentraciones de restos de cetáceo en el sitio Marazzi 32, bahía Inútil, Tierra del Fuego. Informe de avance primer año, proyecto FONDECYT 1020004. MS.

Borrero, L. A.

1985 La Economía Prehistórica de los Habitantes del Norte de la Isla Grande de Tierra del Fuego. Tesis Doctoral inédita. Facultad de Filosofía y Letras, Universidad de Buenos Aires, Buenos Aires. 1990 Fuego-Pagatonian Bone Assemblages and the Problem of Communal Guanaco Hunting. En Hunter of the past, editado por L. B. Davis y B. O. K. Reeves, pp. 373-399. Unwin Hyman, Londres.

1994-1995 Arqueología de la Patagonia. Palimpsesto, Revista de Arqueología 4: 9-69.

2001 Regional Taphonomy: Background noise and the Integrity of the Archaeological Record. En Ethnoarchaeology of Andean South America. Contributions to archaeological Method and Theory, editado por L. A. Kuznar, pp. 243-254. International Monographs in Prehistory. Ethnoarchaeological Series 4. An Arbor.

2013 Estrategias de caza en Fuego-Patagonia. Comechingonia, Revista de Arqueología 17: 11-26.

Borrero, L. A. y N. Franco

1997 Early Patagonian hunter-gatherers: subsistence and technology. Journal of Anthropological Research 53: 219-239.

Bronk Ramsey, C.

2017 Methods for Summarizing Radiocarbon Datasets. Radiocarbon 59 (2): 1809-1833.

Bunn, H. T.

1982 Animal bones and archaeological inference. Science 215: 494-495.

Calás, E.

2009 La subsistencia de los cazadores-recolectores terrestres del Holoceno Medio y Tardío en el norte de Tierra del Fuego. Tesis de Pregrado inédita. Facultad de Ciencias Sociales, Departamento de Antropología, Universidad de Chile, Chile.
Camps-Febrer, $\mathrm{H}$.

1966 Matière et art mobilier dans la Préhistoire nordafricaine et saharienne. Mémoires du Centre de recherches Anthropologie, Préhistoire et Ethnologie des Pays de la Méditerranée Occidentale, París.

Caracotche, M. I., I. Cruz, S. Espinosa, F. Carballo y J. B. Belardi

2005 Rescate arqueológico en el Parque Nacional Monte León (Santa Cruz, Argentina). Magallania 33 (2): 143-163.

Cartajena, M. I.

2007 Una propuesta metodológica para la estandarización de medidas en huesos cortos de camélidos y para la determinación del sexo. Werken 10: 49-62.

Casamiquela, R. M.

1983 La significación del guanaco (Lama guanicoe) en el ámbito pampeano-patagónico: Aspectos cronológicos, ecológicos, etológicos y etnográficos. Mundo Ameghiniano 4: 21-46.

Caviglia, S. y L. A. Borrero

1978 Bahía Solano: su interpretación paleoetnozoológica en un marco regional. Ponencia presentada al IV Congreso Nacional de Arqueología Argentina. San Juan.

Chapman, A.

1986 Los Selk'nam (la vida de los onas). Emecé, Buenos Aires.

Christensen, $M$.

2016 La industria ósea de los cazadores-recolectores: el caso de los nómades marinos de Patagonia y Tierra del Fuego. Colección Poblamiento Humano de FuegoPatagonia. Ediciones Universidad de Magallanes, Punta Arenas.

Costamagno, S., L. Bourguignon, M.- C. Soulier, L. Meignen, C. Beauval, W. Rendu, C. Mussini, A. Mann y B. Maureille

2017 Bone retouchers and site function in the Quina Mousterian: the case of les pradelles (Marillac-Le-Franc, France). En The origins of bone tool technologies: "Retouching the Palaeolithic: Becoming Human and the Origins of Bone Tool Technology", editado por J. M. Hutson, A. García-Moreno, E. S. Noack, E. Turner, A. Villaluenga y S. Gaudzinski-Windheuser, pp. 165-195. Schloss Herrenhause in Hanover, Heilderberg.

De Lamo

1990 Determinación de edad en guanacos (Lama guanicoe Müller) por análisis del desarrollo y desgaste dentario. Veterinaria Argentina VII (69): 621-625. 
De Nigris, $M$.

2004 El consumo en grupos cazadores recolectores. Un ejemplo zooarqueológico de Patagonia Meridional. Sociedad Argentina de Antropología, Buenos Aires.

De Nigris, M. E. y G. L. Mengoni Goñalons

2004 El guanaco como fuente de carne y grasas en Patagonia. En Contra viento y marea. Arqueología de Patagonia, editado por M. T. Civalero, P. M. Fernández y A. G. Guráieb, pp. 467-476. Instituto Nacional de Antropología y Pensamiento Latinoamericano-Sociedad Argentina de Antropología, Buenos Aires.

De Nigris, M. E. y M. P. Catá

2005 Cambios en los patrones de representación ósea del guanaco en Cerro de los Indios 1 (Lago Posadas, Santa Cruz). Intersecciones en Antropología 6: 109-119.

Fiore, D.

2011 Art in time. Diachronic rates of change in the decoration of bone artefacts from the Beagle Channel region (Tierra del Fuego, Southern South America). Journal of Anthropological Archaeology 30: 484-501. 2012 Diseños y tiempos en el arte mobiliar del Canal Beagle (Tierra del Fuego). Una exploración de los ritmos de cambio en la decoración de artefactos óseos. Relaciones de la Sociedad Argentina de Antropología XXXVII (1): 183-206.

Franco, N. V., Cardillo, C., Otaola, C., Arregui, N. y E. Gaal 2007 Tendencias preliminares en el registro arqueológico del curso medio y superior del arroyo El Lechuza, provincia de Santa Cruz, Argentina. Intersecciones en Antropología 8: 271-285.

Franklin, W. L.

1983 Contrasting socioecologies of South America's wild camelids: the vicuña and the guanaco. En Advances in the study of mammalian behavior, editado por J. K. Eisenberg y D. G. Kleiman, pp. 573-629. American Society of Mammalogist, Pennsylvania.

Gecele, P. I. Concha y L. Adaro

1996 Informe relativo a la determinación de rasgos óseos diferenciadores de edad, sexo y función zootécnica en la Ilama. En Determinación de la función zootécnica a través de un enfoque zooarqueológico aplicado a sitios formativos de la Provincia del Loa (II región). Informe de Avance Fondecyt 1950346. MS.

Gusinde, M.

1931 Los indios de la Tierra del Fuego. Vol. I: Los Selk'nam. Centro Argentino de Etnología Americana. Buenos Aires

Hajduk, A. y M. J. Lezcano

2005 Un "nuevo-viejo" integrante del elenco de instrumentos óseos de Patagonia: los machacadores óseos. Magallania 33 (1): 63-80.
Hajduk A., Albornoz, A. y M. J. Lezcano

2004 El Mylodon en el patio de atrás. Informe preliminar sobre los trabajos en el sitio El Trébol, ejido urbano de San Carlos de Bariloche, provincia de Río Negro. En Contra viento y marea. Arqueología de Patagonia, editado por M. T. Civalero, P. M. Fernández y A. G. Guráieb, pp. 715-731. Instituto Nacional de Antropología y Pensamiento Latinoamericano-Sociedad Argentina de Antropología, Buenos Aires.

Hogg, A. G., Q. Hua, P. G. Blackwell, M. Niu, C. E. Buck, T. P. Guilderson, T. J. G. Heaton, J. G. Palmer, P. J. Reimer,

R. W. Reimer, C. S. M. Turney y S. R. H. Zimmerman 2013 SHCal13 Southern Hemisphere Calibration, 0-50,000 Years cal BP. Radiocarbon 55 (4).

Kaufmann, C.

2009 Estructura de edad y sexo en guanaco. Estudios actualísticos y arqueológicos en Pampa Patagonia. Sociedad Argentina de Antropología, Buenos Aires.

Legoupil, D. (Director)

2003 Cazadores-recolectores de Ponsonby (Patagonia austral) y su paleoambiente desde VI al III milenio A.C. / Les chasseurs-cueilleurs de Ponsonby (Patagonie australe) et leur environnement du Vle au Ille mill. av. J. C., Magallania, tirada especial (Documentos). Ediciones Universidad de Magallanes, Punta Arenas.

Loponte, D.

2008 Arqueología del Humedal del Paraná Inferior (Bajíos Ribereños Meridionales). Serie Monográfica I, Instituto Nacinoal de Antropología y Pensamiento Latinoamericano, Buenos Aires.

Lyman, R. L.

1994 Vertebrate Taphonomy. Cambridge University Press, Nueva York.

2008 Quantitative paleozoology. Cambridge University Press, Nueva York.

Mallye, J.-B-, C. Thiébaut, V. Mourre, S. Costamagno, É. Claud y P. Weisbecker

2012 The Musterian bone retouchers of Noisetier Cave: experimentation and identification of marks. Journal of Archaelogical Science 39: 1131-1142.

Massone, M.

1988 Artefactos óseos del yacimiento arqueológico Tres Arroyos (Tierra del Fuego). Anales del Instituto de la Patagonia, serie Ciencias Humanas 18: 107-112. 2017 Fuego, fogones y contextos arqueológicos de los cazadores recolectores tardíos en el norte de Tierra del Fuego. Tesis Doctoral inédita. Facultad de Ciencias Sociales, Universidad Nacional del Centro de la Provincia de Buenos Aires, Olavarría. 
Massone, M. y F. Morello

2007 Los cetáceos en el mundo selk'nam: una evaluación arqueológica. En Arqueología de FuegoPatagonia. Levantando piedras, desenterrando huesos... y develando arcanos, editado por F. Morello, M. Martinic, A. Prieto y G. Bahamonde, pp. 709-721. Ediciones Centro de Estudios del Cuaternario Fuego, Patagonia y Antártica (CEQUA), Punta Arenas.

Massone, M., F. Morello, A. Prieto, M. San Román, F. Martin y P. Cárdenas

2003 Sitios arqueológicos, restos de cetáceos y territorios locales selk'nam en bahía Inútil, Tierra del Fuego. Magallania 31: 45-59.

Mengoni-Goñalons, G. L.

1983 Prehistoric utilization of faunal resources in arid Argentina. En Animals and Archaeology, 1: Hunters and their Prey, editado por J. Clutton-Brock y C. Grigson, pp. 325-335. BAR International Series 163. Archaeopress, Oxford.

1999 Cazadores de guanacos de la estepa patagónica. Sociedad Argentina de Antropología, Buenos Aires.

Mengoni Goñalons, G. L. y M. J. Silveira

1976 Análisis e interpretación de los restos faunísticos de la Cueva de las Manos, Estancia Alto Río Pinturas (provincia de Santa Cruz). Relaciones de la Sociedad Argentina de Antropología X (nueva serie): 261-270.

Mengoni Goñalons, G. L. y M. De Nigris

1999 Procesamiento de huesos largos de guanaco en Cerro de los Indios 1 (Santa Cruz). En Soplando en el viento... Actas de las Terceras Jornadas de Arqueología de la Patagonia, editado por J. B. Belardi, P. M. Fernández, R. A. Goñi, A. G. Guráieb, M. De Nigris, pp. 461-475. Facultad de Humanidades de la Universidad Nacional del Comahue. Neuquén - Buenos Aires.

Mozota Holgueras, M.

2012 The experimental programs to study the bone tools from the Middle Paleolithic hunter-gatherers. En International Conference on Use-Wear Analysis, editado por J. Marreiros, N. Bicho y J. F. Gibaja, pp. 512-520. Cambridge Scholars Publishing, Newcastle upon Tyne. 2015 Un análisis tecno-funcional de los retocadores óseos musterienses del norte de la Península Ibérica, y su aplicación al estudio de grupos neandertales. Munibe Antrolología-Arkeologia 66: 005-021.

Muñoz, A. S.

1997 Explotación y procesamiento de ungulados en Patagonia meridional y Tierra del Fuego. Anales del Instituto de la Patagonia, Serie Ciencias Sociales 25: 201-222.
2000 El procesamiento de guanacos en Tres Arroyos

1, Isla Grande de Tierra del Fuego. En Desde el País de los Gigantes. Perspectivas Arqueológicas de la Patagonia, editado por J. B. Belardi, F. Carballo y S. Espinoza, T. II, pp. 499-517. Universidad de la Patagonia Austral, Río Gallegos.

2002 La explotación de los mamíferos por cazadoresrecolectores terrestres de Tierra del Fuego. Tesis Doctoral inédita. Facultad de Filosofía y Letras, Universidad de Buenos Aires, Buenos Aires.

2008. El procesamiento de los camélidos fueguinos en el pasado. Aspectos metodológicos y resultados alcanzados para el sector atlántico de Tierra del Fuego. En Temas de arqueología. Estudios Zooarqueológicos y Tafonómicos (I), Instituto Nacional de Antropología y Pensamiento Latinoamericano, compilado por A. Acosta, D. Loponte y L. Mucciolo, pp. 77-97. Instituto Nacional de Antropología y Pensamiento Latinoamericano, Buenos Aires.

2012 Guanaco Butchering by Hunter-gatherers from Isla Grande de Tierra del Fuego, Southern Patagonia. En Bones for tools - tools for bones. The interplay between objects and objectives, editado por K. Seetah y B. Gravina, pp. 75-86. McDonald Institute Monographs, Oxford.

Muñoz, S. A. y J. B. Belardi

1998 El marcado perimetral en los huesos largos de guanaco de Cañadón Leona (colección Junius Bird): implicaciones arqueofaunísticas para Patagonia Meridional. Anales del Instituto de la Patagonia, Serie Ciencias Humanas 26: 107-118.

Otaola, C. y N. Franco

2008 Procesamiento y consumo de guanaco en el sitio Chorrillo malo 2, provincia de Santa Cruz, Argentina. Magallania 36 (2): 205-219.

Parker, R. B. y H. Toots

1970 Minor elements in fossil bone. Geological Society of American Bulletin 81: 925-932.

Patou-Mathis, M.

1994 Taphonomie/Bone Modification. Outillage peu élaboré en os et en bois de Cervidés, IV, Artefacts 9 . CEDARC, Treignes.

Patou-Mathis, M. E. (Director) 2002 Fiches typologiques de l'industrie osseuse préhistorique. Cahier X, compresseurs, percuteurs, retouchoirs. Editions de la Société Préhistorique Française, París.

Poplin, F.

1976 A propos du nombre de restes et du nombre $d^{\prime}$ individus dans les échantillons d'ossements. Cahier du Centre de Recherches Préhistoriques 5: 61-74. 
Prieto, A., E. Calás, F. Morello y J. Torres

2007 El sitio arqueológico Myren 2, Tierra del Fuego, Chile. Magallania 35 (2): 89-103.

Raedeke, K. J.

1978 El guanaco de Magallanes, Chile. Su distribución y biología. Corporación Nacional Forestal de Chile, Chile.

San Román, M.

2004 L'exploration des mammifères chez les chasseurs maritimes du site de Bahía Buena: économie des anciens canoeros de Patagonie (Détroit de Magellan). Tesis de Master inédita. Paris I Panthéon Sorbonne, París.

San Román, M., M. Christensen, D. Legoupil, V. Sierpe, F. Martin y F. Morello

2014 Guanacos onboard: Camelid transport and processing by Marine Hunters in Patagonia, Chile. Presentación en el internacional Council for Archaeozoology. San Rafael, Argentina. Póster.

Santiago, F.

2013 La ocupación humana del norte de Tierra del Fuego durante el Holoceno medio y tardío. Editorial Cultural Tierra del Fuego, Ushuaia.

Santiago, F. y M. C. Salemme

2016 Guanaco hunting strategies in the northern plains of Tierra del Fuego, Argentina. Journal of Anthropological Archaeology 43: 110-127.

Santiago, F., N. Pal y M. C. Salemme

2019 Tecnología ósea en el Holoceno tardío de Tierra del Fuego (Argentina): el sitio Las Vueltas 1. Archeofauna. En prensa.

Scheinsohn, V.

1993-1994 Hacia un modelo del aprovechamiento de las materias primas óseas en la isla Grande de Tierra del Fuego (Argentina). Relaciones de la Sociedad Argentina de Antropología XIX: 307-324.

1997 Explotación de materias primas óseas en la Isla Grande de Tierra del Fuego. Tesis Doctoral inédita. Facultad de Filosofía y Letras, Universidad de Buenos Aires, Buenos Aires.

2010 Hearth and bones: Bone raw material exploitation in Tierra del Fuego. BAR International Series 2094. Archaeopress, Oxford.
2013 Bone material and design choices in Southern Patagonia. En From these bare bones, editado por A. Choyke y S. O'Connor, pp. 36-49. Oxbow Books, Oxford.

Sierpe, V.

2015 Atlas osteológico del guanaco (Lama guanicoe). Ediciones Universidad de Magallanes, Punta Arenas. 2018 Los Artiodáctilos y su importancia económica entre los cazadores-recolectores del Holoceno medio y tardío de Fuego-Patagonia, Chile. Tesis Doctoral inédita. Facultad de Facultad de Ciencias Sociales y Jurídicas, Universidad de Tarapacá, Chile.

Sierpe, V., A. Prieto, C. Huidobro y C. Stern 2009 Excavaciones arqueológicas en el sitio "Alero Quemado" (Última Esperanza, Magallanes, Chile). Magallania 37 (2): 177-189.

Silveira, M. J.

1979 Análisis e interpretación de los restos faunísticos de la Cueva Grande del Arroyo Feo (provincia de Santa Cruz). Relaciones de la Sociedad Argentina de Antropología XIII: 229-254.

Torres, J.

2009 La pesca entre los cazadores recolectores terrestres de la Isla Grande de Tierra del Fuego, desde la prehistoria a tiempos etnográficos. Magallania 37 (2): 109-138.

\section{NOTAS}

1.- Para resultados de peces ver Torres (2009).

2.- Instituto de la Patagonia, Universidad de Magallanes, Punta Arenas, Chile.

3.- Cálculo realizado en base a la recomposición o ensamblaje del atlas.

4.- Las cúpulas de hundimiento expresadas aquí corresponden a los estigmas producidos sobre la superficie de la materia ósea producto del aplastamiento y desprendimiento de parte de la superficie, debido a un traumatismo realizado sobre el hueso por el choque con un material duro, en este caso, retoque de material lítico. Para más detalle, ver Patou-Mathis (2002). 
240 | V. Sierpe et al. - Intersecciones en Antropología 20 (2), agosto-diciembre. 2019. ISSN-e 1850-373X 